\title{
The 2015 guidelines for idiopathic pulmonary fibrosis: an important chapter in the evolution of the management of patients with IPF
}

\author{
Kevin C. Wilson ${ }^{1,2}$ and Ganesh Raghu ${ }^{3}$ \\ Affiliations: ${ }^{1}$ American Thoracic Society, New York, NY, USA. ${ }^{2}$ The Pulmonary Center, Boston University \\ Medical Center, Boston, MA, USA. ${ }^{3}$ Center for Interstitial Lung Diseases, University of Washington Medical \\ Center, University of Washington, Seattle, WA, USA.
}

Correspondence: Ganesh Raghu, Center for Interstitial Lung Diseases, University of Washington Medical Center, 1959 NE Pacific, Campus Box 356175, Seattle, WA 98195, USA. E-mail: graghuduw.edu

@ERSpublications

2015 guidelines for treatment of IPF empowers the clinician and patient to make individualised treatment decisions http://ow.ly/R37nz

During the past 15 years, clinical practice guidelines have evolved from the consensus-based opinions of experts to evidence-based documents developed using a rigorous systematic approach. In 2000, a selected panel of international experts in the field of interstitial lung diseases developed a guideline for the diagnosis and management of idiopathic pulmonary fibrosis (IPF) [1]. The guideline was based upon the consensus opinions of the few expert panel members; the panel members' decisions were not informed by a systematic review of the literature, meaning that there was no formal synthesis of the evidence or appraisal of the quality of the evidence. Despite these limitations, the guideline was considered state of the art for the time. It provided important direction to clinicians, including the first concrete definition for IPF, and provoked new studies. During the following decade, abundant evidence accumulated at a rapid rate, making it difficult for the average clinician to synthesise, interpret, and apply the evidence to their clinical practice. To address this, and to improve upon the previous document, the IPF guideline was updated in 2011 using an evidence-based approach for the first time [2]. The updated guideline redefined IPF using precise diagnostic criteria based on the clinical, radiological and histopathological features of IPF; it also it provided evidence-based treatment recommendations for the first time in the field of IPF.

The recently published 2015 American Thoracic Society (ATS)/European Respiratory Society (ERS)/ Japanese Respiratory Society (JRS)/Latin American Thoracic Society (ALAT) clinical practice guidelines on the treatment of IPF (herein, called the "2015 IPF guidelines") [3] are a treatment-specific update of the prior versions of the IPF guidelines $[1,2]$. These guidelines were developed using a process that included stricter conflict of interest management, a more rigorous methodological approach, and broader stakeholder involvement than previous versions. The goal of the escalated rigour was to produce guidelines that were less biased, more transparent, and more trustworthy than prior guidelines [4].

The panel systematically reviewed the body of evidence for 12 treatment questions related to IPF. Eight new or revised recommendations were rendered, two recommendations were left unchanged, and the formulation of recommendations for two questions was deferred (table 1). Among the most notable new or revised recommendations, compared with the 2011 guidelines, are a strong recommendation against using the combination of prednisone, azathioprine and $\mathrm{N}$-acetylcysteine, a conditional recommendation for the use of nintedanib, and a conditional recommendation for the use of pirfenidone. The

Received: Aug 122015 | Accepted: Aug 122015

Conflict of interest: Disclosures can be found alongside the online version of this article at erj.ersjournals.com

Copyright $\odot$ ERS 2015 
TABLE 1 Comparison of recommendations in the 2015 and 2011 idiopathic pulmonary fibrosis (IPF) guidelines

Agent 2015 guideline 2011 guideline

New and revised recommendations

Anticoagulation (warfarin)

Combination prednisone + azathioprine + $N$-acetylcysteine

Selective endothelin receptor antagonist (ambrisentan)

Imatinib, a tyrosine kinase inhibitor with one Strong recommendation against use ${ }^{\#}$ target

Nintedanib, a tyrosine kinase inhibitor with multiple targets

Pirfenidone

Dual endothelin receptor antagonists (macitentan, bosentan)

Phosphodiesterase-5 inhibitor (sildenafil)

Unchanged recommendations

Antiacid therapy

$N$-acetylcysteine monotherapy

Anti-pulmonary hypertension therapy for IPF-associated pulmonary hypertension

Lung transplantation: single versus bilateral lung transplantation
Strong recommendation against use ${ }^{\#}$

Strong recommendation against use ${ }^{+}$

Strong recommendation against use ${ }^{+}$

Conditional recommendation for use

Conditional recommendation for use

Conditional recommendation against use ${ }^{+}$

Conditional recommendation against use $\mathrm{A}^{\#}$

Conditional recommendation for use

Conditional recommendation against use ${ }^{+}$

Reassessment of the previous recommendation was deferred

Formulation of a recommendation for single versus bilateral lung transplantation was deferred
Conditional recommendation against use Conditional recommendation against use ${ }^{+}$

Not addressed

Not addressed

Not addressed

Conditional recommendation against use Strong recommendation against use ${ }^{\#}$

Not addressed

Conditional recommendation for use

Conditional recommendation against use ${ }^{+}$

Conditional recommendation against use

Not addressed

${ }^{\#}:+++-$, moderate confidence in effect estimates; ${ }^{\text {I: }}+---$, very low confidence in effect estimates; ${ }^{+}:++--$, low confidence in effect estimates. Reproduced from [3] with permission from the publisher.

recommendation against the combination regimen is the consequence of new findings that the regimen increases mortality and hospitalisations, findings that were published after the prior version of the IPF guidelines [5]. The recommendations for nintedanib and pirfenidone represent the first time that the panel has recommended an IPF-specific therapy. In a well-defined population of patients with IPF, both nintedanib [6,7] and pirfenidone [8-11] reduce disease progression, as indicated by a decreased rate of forced vital capacity decline over a year and a suggested decrease in clinically meaningful end-points, such as mortality and acute exacerbations, when the data were pooled.

Strict management of potential conflicts of interest and an independent evidence synthesis were two aspects of the development of the 2015 IPF guidelines that were designed to mitigate bias. The conflict of interest rules established for the IPF guidelines required that at least half of the co-chairs had no conflicts of interest related to the content of the guidelines and, similarly, that at least half of the panel had no conflicts of interest related to the content of the guidelines. In addition, the rules stipulated that panellists could not participate in decision-making (i.e. formulating or grading recommendations) related to their conflicts of interest. The co-chairs elected to shield the guidelines even further from potential accusations of bias by broadening the recusals so that panellists with a conflict of interest could not participate in any decision-making, regardless of whether the decision-making was or was not related to their conflict. It is important to note that all panellists, including those recused from decision-making, were allowed to discuss the evidence; this was necessary to ensure that the appropriate expertise was available to inform the decision-makers.

During the panel's deliberations, adherence to the conflict of interest rules was strict. The individuals who were recused were respectful of the rules, and in the few instances when they began to make a comment during the decision-making phase they were immediately cut-off by the co-chairs and reminded of the rules. Following the deliberation of the recommendations by the individuals who did not have any conflicts of interest, the individuals who were recused due to potential conflicts of interest were specifically asked what their recommendation would have been if they were allowed to make recommendations. The overall consensus among those who were recused from decision-making was that the process in general worked well and was a clear improvement compared with prior guidelines. The same recommendations would have resulted with their participation in the decision-making, with one exception; most of the recused panellists would not have made a recommendation for or against anti-acid therapy due to the lack of randomised trial data.

There are several acceptable approaches when data are scarce. Guideline developers may opt to make no recommendation, may make a recommendation for research, or may make a recommendation based on 
low or very low quality evidence. In these guidelines, the panellists who were allowed to participate in decision-making chose the third option, whereas most of the recused experts would have chosen the first option. The reason for the different decisions were that the non-conflicted panellists felt that making a recommendation would be more helpful to clinicians even if it was based upon very low quality evidence, whereas the conflicted panellists were concerned that the anti-acid recommendation would be perceived as equivalent to other conditional recommendations that were based upon better evidence. It should be emphasised that recommendations of similar strength should not be interpreted as achieving the same net benefit or harm; each recommendation's strength is the net result of multiple factors and, therefore, there may be different reasons that two recommendations are rated as having the same strength.

Conflict of interest management was not the only way that potential bias was mitigated. For each clinical question the systematic evidence synthesis (i.e. identifying, selecting, summarising and appraising the body of evidence) was conducted by a team of trainees from McMaster University with the oversight of the ATS methodologist and one of the co-chairs who had no conflicts of interest. The trainees had no conflicts of interest and no intellectual investment in IPF; therefore, they were not swayed by a particular viewpoint for any clinical question.

To keep the mission of guideline-development patient focused, a patient representative was included on the guideline panel. William C. Cunningham was actively involved in all stages of guideline development, including providing input and approval of the first version of the manuscript that was submitted to external review; unfortunately, he passed away prior to publication of the guidelines. Dedication of the guidelines to William C. Cunningham is indicative of the panel's appreciation of his contributions. His input was absolutely essential. Formulating a recommendation requires that the desirable consequences of an intervention (i.e. benefits) be weighed against the undesirable consequences (i.e. side-effects, costs, burdens and resource use). When these competing factors are finely balanced, insight from patients about the relative importance of various benefits, side-effects and burdens is enormously helpful because patients are in a better position to make such assessments than clinicians. For the vast majority of treatment questions considered, the panellists requested William C. Cunningham's opinion about the importance of one or more outcomes, in order to help make their decision about whether or not to recommend an intervention.

For conditional recommendations, especially those based on a low or very low certainty of evidence, the 2015 IPF guidelines emphasise the importance of shared decision-making between clinicians and patients in considering all of the factors described above, as well as the individual patient's values and preferences. The 2015 IPF guidelines provide a scientific rationale that empowers patients, clinicians, and stakeholders to better understand how the recommendations were formulated and to better apply them to their specific clinical practices and situations.

While the rationale for the recommendations made in the 2015 IPF guidelines has been discussed [3] and the recommended IPF-specific therapies have offered hope for patients with IPF for the very first time, there are several unknowns and questions that clinicians and patients considering IPF-specific therapy will need to be aware of and that need to be addressed in future studies [12]. In addition, there are other IPF-related questions that the co-chairs hope to answer in future iterations of the guidelines. Among the most urgent questions are whether anti-pulmonary hypertension therapy is indicated for IPF-associated pulmonary hypertension, and whether single or bilateral lung transplantation should be performed. While the association between IPF and gastro-oesophageal reflux is well known, it is unclear whether IPF is a result or cause of the gastro-oesophageal reflux; studies to answer this question are important because the answer may affect the role of anti-acid treatment for presumed abnormal acid gastro-oesophageal reflux in patients with IPF (i.e. patients with IPF who do not have any symptoms of gastroesophageal reflux) or surgical therapies for gastro-oesophageal reflux in patients with IPF. Studies looking at the effects of combination therapies are needed to determine if agents that act via different mechanisms have synergistic effects. The identification of subgroups of IPF patients (i.e. phenotypes) that differentially respond to therapies may lead to a more personalised and effective approach to treating patients with IPF. Finally, it should be noted that the 2015 IPF guidelines updated only the treatment aspects of the 2011 IPF guidelines. There is also a need to update the diagnostic criteria for IPF; until such an update is carried, however, diagnosis of IPF should continue in accordance with the 2011 IPF guidelines.

In essence, the 2015 IPF guidelines represent an important chapter in the evolution of the management of patients with IPF. We hope that this chapter provides recommendations that make patients' lives better, by either recommending interventions that result in benefits or recommending against interventions that are likely to cause harm. The end result, we hope, will be improved outcomes that are meaningful for patients with IPF and ultimately the cure of a debilitating and deadly disease. 


\section{References}

1 American Thoracic Society, European Respiratory Society. Idiopathic pulmonary fibrosis: diagnosis and treatment. international consensus statement. Am J Respir Crit Care Med 2000; 161: 646-664.

2 Raghu G, Collard HR, Egan JJ, et al. An official ATS/ERS/JRS/ALAT statement: idiopathic pulmonary fibrosis: evidence-based guidelines for diagnosis and management. Am J Respir Crit Care Med 2011; 183: 788-824.

3 Raghu G, Rochwerg B, Zhang Y, et al. An Official ATS/ERS/JRS/ALAT clinical practice guideline: treatment of idiopathic pulmonary fibrosis. Am J Respir Crit Care Med 2015; 192: e3-e19.

4 Graham R, Mancher M, Miller Wolman D, et al., eds. Clinical Practice Guidelines We Can Trust. Washington, National Academies Press (US), 2011.

5 Raghu G, Anstrom KJ, King TE Jr, et al. Prednisone, azathioprine, and $\mathrm{N}$-acetylcysteine for pulmonary fibrosis. N Engl J Med 2012; 366: 1968-1977.

6 Richeldi L, Costabel U, Selman M, et al. Efficacy of a tyrosine kinase inhibitor in idiopathic pulmonary fibrosis. N Engl J Med 2011; 365: 1079-1087.

7 Richeldi L, du Bois RM, Raghu G, et al. Efficacy and safety of nintedanib in idiopathic pulmonary fibrosis. $N$ Engl J Med 2014; 370: 2071-2082.

8 Azuma A, Nukiwa T, Tsuboi E, et al. Double-blind, placebo-controlled trial of pirfenidone in patients with idiopathic pulmonary fibrosis. Am J Respir Crit Care Med 2005; 171: 1040-1047.

9 Taniguchi H, Ebina M, Kondoh Y, et al. Pirfenidone in idiopathic pulmonary fibrosis. Eur Respir J 2010; 35: 821-829.

10 Noble PW, Albera C, Bradford WZ, et al. Pirfenidone in patients with idiopathic pulmonary fibrosis (CAPACITY): two randomized trials. Lancet 2011; 377: 1760-1769.

11 King TE Jr, Bradford WZ, Castro-Bernardini S, et al. A phase 3 trial of pirfenidone in patients with idiopathic pulmonary fibrosis. N Engl J Med 2014; 370: 2083-2092.

12 Raghu G, Selman M. Nintedanib and pirfenidone. New antifibrotic treatments indicated for idiopathic pulmonary fibrosis offer hopes and raises questions. Am J Respir Crit Care Med 2015; 191: 252-254. 